\title{
La democrazia e l'educazione, valori cooperativi per i giovani $^{1}$
}

\author{
Vega María Arnáez Arce \\ Alberto Atxabal Rada \\ Università di Deusto, Bilbao
}

Recibido: 10-03-2014

Aceptado: 03-05-2014

Sommario: I. Introduzione. II. La democrazia come valore cooperativo. 2.1. II principio de la gestione/governance democratica. 2.2. II principio della partecipazione economica dei soci. III. La istruzione, la formazione e I' informazione. IV. I giovani e le cooperative. 4.1. L'ACl e i giovani. 4.2. II raggiungimento dell'occupazione attraverso le cooperative. 4.3. La formazione e l'educazione dei giovani. 4.4. La partecipazione dei giovani nella cooperativa. V. Bibliografia.

Astratto: II titolo della presentazione si occupa delle relazioni tra cooperative e giovani e, più specificamente, tra i valori cooperativi e i giovani. Parleremo dei rapporti tra i valori cooperativi e i giovani, alla cui base stanno i valori di democrazia ed educazione. Cercheremo, infatti, di evidenziare la diversa natura della cooperativa rispetto ad altre forme di impresa perchè, esse portano nel loro DNA la democrazia e I' educazione, ed entrambi dovrebbero essere, allo stesso tempo, un'attrazione affinché i giovani possano avvicinarsi al mondo cooperativo.

Parole chiave: democrazia, educacione, giovani, cooperative.

Abstract: The title of the presentation deals with the relations between cooperatives and young people and, more specifically, between the co-operative values and young people. We will discuss the relationship between the cooperative values and young people, as long as they are co-operative values of democracy and education. We will try, in fact, to highlight the diverse nature of the cooperative compared to other forms of business because they carry in their DNA democracy and education, and both should be at the same time, an attraction for young people to get closer to the cooperative world .

Key-words: democracy, education, young people, cooperatives.

1 Presentazione consegnata in LUMSA di Roma il 6 maggio 2014 nel convegno dal titolo «Quale diritto per un'economia sociale?». 
Buonasera.

Vorremo, prima di tutto, ringraziare la LUMSA per averci offerto l'opportunità di condividere questa esperienza con illustri professori, esperti nell'ambito dell'Economia Sociale. Ma soprattutto vogliamo ringraziare il Professor Antonio Fici per il suo gentile invito a venire a Roma e per potere esporre alcune idee sui valori cooperativi e i giovani che spero siano $d^{\prime}$ interesse, in un'area di importanza vitale per le nuove generazione e per l'economia in generale.

Il titolo della presentazione che condivido con la Professoresa Arnáez si occupa delle relazioni tra cooperative e giovani e, più specificamente, tra i valori cooperativi e i giovani. Tuttavia, la nostra participazione sarà molto meno ambiziosa di quello che il titolo promette. Parleremo dei rapporti tra i valori cooperativi e i giovani, alla cui base stanno i valori di democrazia ed educazione.

Cercheremo, infatti, di evidenziare la diversa natura della cooperativa rispetto ad altre forme di impresa perchè, esse portano nel loro DNA la democrazia e l' educazione, ed entrambi dovrebbero essere, allo stesso tempo, un'attrazione affinché i giovani possano avvicinarsi al mondo cooperativo.

\section{Introduzione}

L'Assemblea Generale delle Nazioni Unite ha proclamato il 2012 «Anno Internazionale delle Cooperative». A seguito di questa dichiarazione, I'Assemblea Generale della Alleanza Cooperativa Internazionale $(\mathrm{ACl})$ tenuta a Manchester nel ottobre 2012 ha approvato un piano per un Decennio Cooperativo incoraggiando alle cooperative il coinvolgimento nel settore dei giovani offrendo loro uno spazio per favorire il loro sviluppo personale e professionale. Questa proposta ci permette d'indagare sui valori cooperativi che attirano i giovani rispetto il movimento cooperativo. Tra questi valori, la democrazia, in particolare, gioca un ruolo convergente come valore differenziale della Cooperativa e come valore ideale della gioventù.

I cooperativisti hanno qualcosa da dire di fronte al I'avversa situazione economica che stiamo affrontando oggi. I tagli di tanti Governi alla spesa pubblica e sociale hanno provocato e continuano a provocare le disuguaglianze mentre, contemporaneamente, si producono cambiamenti sostanziali nel potere economico.

Ci chiediamo se c'è qualche alternativa per il prevalente modello economico, cioé, il modello capitalistico. Le cooperative possono por- 
tare un raggio di speranza e di chiarezza ai cittadini del mondo e ai giovani in particolare. Noi continuiamo a chiederci: perché la cooperativa? Che cosa ha di speciale? Tra i diversi modelli d'impresa, solo le cooperative controllano democraticamente le loro risorse economiche. La cooperativa è la formula più autentica e diretta dell'aplicazzione della democrazia nel mondo dell'azienda. II modello cooperativo è un modo commercialmente efficace ed efficiente di agire l'attività di impresa, che prende in considerazione, in maniera elevata, i bisogni umani e i valori nel processo decisionale. Inoltre, la cooperativa, supponendo la bontà dei suoi postulati, educherà e formerà a questi valori e a questi principi i suoi soci e informerà le autorità pubbliche e la società in generale circa la necessità di rispettare questi valori come un bene comune per tutta la società. Vediamo, quindi, che cosa sono le cooperative e come possono essere attraenti per i giovani del nostro tempo.

\section{La democrazia come valore cooperativo}

In questo contesto, è necessario preservare la democrazia come valore cooperativo che consenta di distinguere le cooperative dal resto delle organizzazioni economiche, poiché altri valori, sia in forma esplicita che implicita, potrebbero essere assunti per le imprese capitalistiche convenzionali. La struttura delle cooperative plasma la democrazia come una norma di comportamento e di caratteristica differenziata tra le altre aziende. Non basta la forma, deve esserci la volontà di coinvolgere tutte le parti.

La democrazia come valore cooperativo ci impone di ripensare alle grandi domande che riguardano gli esseri umani: quali sono i limiti della democrazia? come possono organizzare gli uomini e le donne le loro società affinché ci sia più equita? Come può cambiare l'economia in modo che sia efficace ed etica allo stesso tempo? Quali sono le richieste giuste dei lavoratori? Come remunerare il capitale? Come si può organizzare l'economia per produrre abbastanza beni a prezzi equi e con buona qualità per soddisfare le esigenze di tutti? Come si possono costruire comunità migliori? Cooperative e cooperatori non possono risolvere tutti questi problemi da soli, ma possono contribuire molto nel farlo.

E le cooperative di lavoro associato tanto più, perché hanno all' interno delle loro strutture e delle loro filosofie alcune delle risposte più persusive rispetto alla grande domanda sollevata dall'industrializzazione: come garantire che i lavoratori possano godere della dignità a cui il loro lavoro da diritto? La prospettiva della cooperativa di lavoro promuove anche il fatto che altri tipi di cooperative possano considerare con più attenzione come vedono e come trattano i loro dipendenti. 
La democrazia è uno dei valori inerenti alla natura delle cooperative, da una doppia dimensione: verso l'interno della cooperativa, dove la proprietà, il controllo e la gestione delle cooperative è effettuata dai soci in maniera democratica; e all'esterno della cooperativa, dove il socio cooperativo partecipa all'economia e organizza i fattori della produzione attraverso una società basata sulla persona e non sul capitale. Nelle parole dell' ACl, fomentare questo spirito democratico è un compito senza fine, difficile, prezioso e addiritura, indispensabile.

La democrazia si incardina su due principi, vale a dire, sul principio della governance democratica e sul principio della partecipazione economica dei soci.

\subsection{Il principio de la gestione/governance democratica}

Il principio della governance democratica è, quindi, un principio che ha una notevole importanza, soprattutto per le cooperative composte principalmente da soci-lavoratori. Se la persona è libera, e i membri di una cooperativa sono persone, si deve permettere a tutti di partecipare alla cooperativa con processi decisionali e identica libertà. E in questo che si differisce sostanzialmente dalla società capitalista. Nella cooperativa s'intende che, attraverso una gestione democratica, la persona si senta perfettamente integrata e identificata con l'azienda dove lavora. È il caso, ad esempio, delle cooperative di Mondragon, gruppo composto, principalmente, da cooperative di lavoro associato o di produzione.

Ma non dobbiamo dimenticare che la cooperativa è una società nel senso che è un'entità organizzata che opera sul mercato e quindi dovrebbe servire i membri efficacemente ed efficientemente. In questa prospettiva, i soci responsabili della gestione delle cooperative mantengono i loro posti in fideicomiso per il profitto immediato e a lungo termine.

Le cooperative non appartengono più ai dirigenti eletti ma ai dipendenti. Perciò i dirigenti risponderano delle loro azioni d'avanti ai soci durante il loro mandato.

D'altra parte, non è difficile vedere come il valore della democrazia tra i soci richieda una sorta di strategia nei rapporti, e una politica di distribuzione delle informazioni. I soci hanno il diritto di partecipare, di essere informati, di essere ascoltati e di essere coinvolti nel processo decisionale. Questa democrazia nella gestione delle cooperative di primo grado si manifesta nella distribuzione dei voti: un socio, un voto.

Questa regola di un voto per ogni socio è minata dal quadro legislativo delle cooperative di primo grado quando se introduce un voto ponderato. 
Ugualmente, I'attuazione pratica di tali diritti in modo equo diventa, a volte, un compito difficile nelle grandi cooperative o nelle cooperative di secondo o di ulteriore grado. Inoltre, la necessità per le società cooperative di accedere al credito ha portato il legislatore a regolamentare la possibilita in modo che il processo decisionale sia proporzionale al capitale sottoscritto dai soci capitalisti della cooperativa, come nel caso delle cooperative miste, che presupone un'altra distorsione del principio democratico di partecipazione.

Tuttavia, purchè si tratti di una impresa di proprietà comune e democraticamente controllata. Nelle cooperative il controllo si distribuisce sia su base democratica che su un fattore di differenziazione rispetto alla società capitalistica o ad altre organizzazioni controllate dai governi. La prevalenza della persona sul capitale è una conseguenza immediata e diretta dei principi giuridici di democrazia e di partecipazione.

\subsection{Il principio della partecipazione economica dei soci}

Le cooperative operano affinché il capitale sìa il "servo» e non il «padrone» dell'organizzazione. Senza dubbio, la subordinazione del capitale nella cooperativa si rivela attraverso I'interesse limitato al capitale. In contrasto con la società capitalista, la cooperativa non concede al capitale diritti politici. II capitale è un mero strumento per il raggiungimento del fine sociale della cooperativa: un chiaro esempio del carattere personale della società cooperativa.

Ovviamente, i membri devono contribuire, con un certo capitale, alla cooperativa per acquisire tale condizione. Inoltre va notato che la cooperativa è definita come una società in cui la proprietà è esercitata democraticamente. Questo significa che i cooperativisti possono individualmente essere proprietari delle parti che hanno firmato, ma non sono proprietari individuali della cooperativa o del suo attivo. Ecco qualle è la differenza tra il valore di una parte della cooperativa e il valore di un' azione in una società di capitale.

La prevalenza della persona sul capitale, latente nella democrazia come un valore, ha generato l'uguaglianza di tutti i membri nel diritto al voto. Un membro, un voto. II metro per misurare la partecipazione nella cooperativa non viene determinato dalla quantità di capitale, ma si concede lo stesso valore al parere di tutte le persone, indipendentemente dal volume della loro partecipazione al capitale.

Questa idea si è voluta applicare alla participazione economica dei soci nel capitale, anche se non è facile l'applicazione in tutte quelle 
cooperative che hanno una certa dimensione perchè le leggi del mercato pressano affinchè i lavori più qualificati vengano pagati in base alla professionalita.

Per esempio, nelle cooperative di lavoro associato le differenze salariali non sono così drammatiche come nella società capitalista. Questo principio viene chiamato "solidarietà retributiva». Ma ciò non esclude che essista una relazione, che è in aumento da 1 a 3, da 1 a 4 o da 1 a 5 tra i soci che più ricevono per le loro operazioni nella cooperativa di fronte a quelli che ricevono meno.

In definitiva, i soci contribuiscono equamente al capitale delle loro cooperative e lo gestiscono in modo democratico. Almeno parte di tale capitale è solitamente proprietà comune della cooperativa. I membri ricevono una compensazione, se c'è, limitata al capitale che è stato versato per essere socio.

\section{La istruzione, la formazione e l' informazione}

Abbiamo già sottolineato l'importanza del movimento cooperativo come un esempio di partecipazione e gestione democratica, basata sui valori come la solidarietà, la democrazia, I'uguaglianza, I'equità, I'autonomia e la responsabilità personale, al punto di poter dire che ciò che distingue le entità cooperative non è altro che lo scopo sociale o la specifica attività svolta, ma la sua essenza e i principi guida e ispiratori che includono l'istruzione, la formazione e l' informazione.

Fin dalle sue origini, il movimento cooperativo ha avuto un impegno chiaro e fermo con l'educazione. Non dobbiamo dimenticare che lo sviluppo del modello di cooperazione di Rochdale e il suo funzionamento sono stati il risultato diretto di un processodi Educazione-Aprendistato.

Tanto che questo principio è stato raccolto nell' elenco dei principi cooperativi, fin dall' origine di Rochdale, consolidandosi, sempre più nel tempo, in tutte le revisioni fatte dall'Alleanza Cooperativa Internazionale. A nostro parere, ciò è dovuto all'importanza dell'educazione come strumento trasformatore della società, nel suo complesso e, allo stesso tempo delle vite degli esseri umani, individualmente considerati.

E così come ha dichiarato esplicitamente I'Alleanza Cooperativa Internazionale quando ha definito l'educazione sia come: "la volontà di condividere esperienze, imparando dai succesi e dai fallimenti» sia come elemento che ha transformato in mezzo secolo di sperimentazione, la cooperazione come modello di successo, tanto da essere imitato poi in tutto il mondo». 
Così, nel 1995, la dichiarazione dell'Alleanza Cooperativa Internazionale sull'identità cooperativa ha fatto rifferimento al quinto principio di educazione, di formazione e di informazione nei seguenti termini:

«Le cooperative forniscono istruzione e formazione ai propri membri, ai rappresentanti eletti, diretti e ai lavoratori affinchè possono contribuire efficacemente allo sviluppo delle loro cooperative. Essi devono informare il grande pubblico, soprattutto i giovani e i leader di opinione, sulla natura e sui benefici della cooperazione».

Così come afferma I'Alleanza nella dichiarazione, in questo quinto principio si sottolinea l'importanza e la rilevanza dell'educazione e della formazione cooperativa, sia verso all'interno sia verso esterno.

Verso I'interno della cooperativa e del proprio movimento, l'educazione tende più che alla semplice distribuzione di informazioni o la promozione dell'utente. Questo tipo di formazione comporta l'impegno delle menti dei soci e dei lavoratori in modo che capiscano bene la complessità e la ricchezza del pensiero e dell' azione cooperativa.

In linea con Martínez Charterina, possiamo dire che l'istruzione è la regola d'oro della cooperativa, che permette ai soci e ai menbri delle cooperative di capire quali sono i fondamenti e il senso delle cooperative, i valori e i principi, il ruolo che ha sviluppato il movimento cooperativo nel corso della storia, così come i diritti e le responsabilità. Tutto questo, con l'obiettivo che i soci cooperativisti contribuiscano alla promozione della pace, alla evoluzione e allo sviluppo del movimento cooperativo.

Allo stesso tempo, nello sviluppo e nella attuazione del principio cooperativo, si aggiungerano altri aspetti della formazione professionale, che interessano non solo i soci ma, anche i dirigenti e i lavoratori, aspetti fondamentali per il buon funzionamento della azienda cooperativa.

In questo senso, l'educazione e la formazione offrono ottime opportunità affinchè i gestori, e i leader cooperativi possano capire i bisogni dei soci, in modo tale che siano in grado di valutare costantemente le attività della cooperativa e suggerire modi per migliorare le operazioni o fornire nuovi servizi.

Per quanto riguarda l'educazione dei soci vengono messi a disposizione dei mezzi dei canali e degli strumenti di conoscenza per aproffondire nella identità dei valori e principi condivisi con il movimento mondiale di cui la cooperativa fa parte.

L'obiettivo o lo scopo finale di questa prima espressione del principio di educazione è che i soci capiscano il significato, la portata dei diritti e le responsabilità inerenti alla appartenenza alla cooperativa e che, allo stesso tempo, si possano comprendere, posizionandosi e agendo 
come membri attivi e informati. Solo così assumerano l'identità, la visione e la missione delle loro rispettive cooperative.

D'altra parte, in relazione ai rappresentanti eletti di ogni cooperativa, l'educazione ha un obiettivo o un punto di partenza diverso: la formazione che constribuisca a una buona gestione, in definitiva, a un buon governo delle cooperative, da quelle più piccole a quelle più grandi con una struttura piu complessa, dato che il succeso o il fallimento dipende in un alto grado delle decisioni prese dai representanti eletti che si occupano della gestione. Pertanto, capiamo che, nella misura in cui i dirigenti sono dotati di conoscenze, di competenze e di abilità, migliori saranno le prospettive di successo e di sviluppo nell'interesse della cooperativa.

Infine, l'istruzione - formazione dei dirigenti e dei dependenti delle cooperative tende a far comprendere che occorre interiorizzare sia le specialità e le peculiarità proprie, sia i bisogni e le aspirazioni dei suoi membri nei confronti di altre societa.

Questo è particolarmente importante, come ha segnalato I'Alleanza Cooperativa Internazionale nell'ambito del Piano per un Decennio Cooperativo, rispetto a quelli che provengono da società «quotate» in cui le esigenze degli azionisti sarano, senza dubbio, sostanzialmente diverse dalle esigenze dei membri di una cooperativa.

D'altra parte, volgendo uno sguardo "ad extra», la presenza delle cooperative nella società e la loro aspirazione a promuovere e a diffondere la convinzione circa la bontà del sistema e i benefici della cooperazione, mette in evidenza il bisogno d'investire e lavorare nella difussione dei valori e dei principi del movimento cooperativo nella società in generale, nei leader in particolare e sopratutto nei giovani.

In modo che le opportunità per lo sviluppo delle cooperative si possano sfruttare di più, l'educazione, deve incoraggiare e promuovere programmi di formazione nelle scuole e nelle università che vengano a contribuire all'approfondimento della conoscenza del movimento cooperativo e, allo stesso tempo, alla diffusione tra i cittadini attraverso i mezzi di comunicazione.

\section{I giovani e le cooperative}

Come è stato accennato in precedenza, il coinvolgimento della persona del socio constituisce uno degli aspetti basilare del movimento cooperativo fin dalle sue origini. Questa preoccupazione per la persona non può essere limitata all'interno della cooperativa. Necessariamente,questo atteggiamento di rispetto per i valori che si 
genera dentro deve avere la sua influenza pubblica ed estendersi ai non-soci e a i giovani.

Il coinvolgimento dei giovani nella cooperativa si verifica principalmente in due modi: in primo luogo, attraverso la creazione di cooperative di giovani e in secondo luogo, attraverso gli sforzi di cooperative create per incoraggiare la partecipazione dei giovani.

\subsection{L'ACl e i giovani}

La Alleanza Cooperativa Internazionale vuole attirare i più giovani verso il mondo cooperativo

come garanzia del successo dei suoi postulati e come rinnovo generazionale dei soci cooperativi attuali. Afferma l'ACl che gran parte della vitalità del movimento cooperativo si otterrà dal coinvolgimento dei giovani.

II Piano per un Decennio Cooperativo dell'ACI tende a focalizzare l'attenzione specifica e diretta tra i giovani e adulti, studiando i meccanismi per creare e mantenere relazioni, valutando se si possono adattare i tradizionali meccanismi di partecipazione e relazione. Le giovani generazioni sono un grado di mettere in atto i propri meccanismi di collaborazione rapportandoli con quelli già stabiliti, per sucessivamente integrarli? Le cooperative offrono i punti di accesso più adatti per i giovani? Essi sono disposti a creare uno spazio o una piattaforma che permetta loro di cambiare il futuro? Usano il linguaggio appropriato?

$\mathrm{L}^{\prime} \mathrm{ACl}$ è consapevole che per atrarrere i giovani al movimento cooperativo deve essere presentato come una forma d'azienda sufficientemente attraente per loro, un posto dove si offrono prosprettive per il fututo. A tale scopo, l'ACl vuole evidenziare che cosa ha di particolare una cooperativa, il suo aspetto idealistico e la sua importanza sociale. I valori e principi cooperativi vengono presentati come la forma principale per collegare i giovani, che potrano trovare non solo le risposte a i loro ideali in un mondo migliore, ma anche una valida causa per cui lavorare e per cui combattere.

Tuttavia, i giovani di oggi non sono quelli di quarant'anni fa, e i loro valori stanno cambiando nel tempo. Possiamo parlare anche di contravalori o di valori negativi dei giovani: la difficoltà che hanno per portare avanti impegni duraturi o la prevalenza dell'individualismo sui valori della solidarietà. Analogamente, ci sono giovani che preferiscono una maggiore partecipazione in altri gruppi di volontariato: ecologia, benessere o attività ricreative. Ora, non tutti i giovani sono uguali e 
non tutti saranno cooperativi. Ovviamente, un giovane uomo si avvicinerà al mondo cooperativo nella misura in cui i suoi valori sarano simili a quelli delle cooperative.

La cooperativa appare come una soluzione adeguata ai problemi della gioventù. L'aspirazione del movimento cooperativo, per migliorare la società e costruire un mondo migliore attraverso gli ideali di pace, di uguaglianza e di democrazia, è senza dubbio un importante contributo agli interessi dei giovani e alle loro condizioni di vita e di futuro.

\subsection{Il raggiungimento dell'occupazione attraverso le cooperative}

Ci sono questioni di giustizia sociale, come la situazione dei giovani nel mercato del lavoro, che le cooperative hanno cercato di risolvere storicamente e che dovrebbero continuare a farlo. In molti paesi del Sud, c'è un'eccedenza di giovani, uomini e donne, in cerca di occupazione, seriamente preoccupati per come organizzeranno la loro vita. Nella maggior parte dei paesi del Nord, le possibilità di pieno d'impiego diminuiscono di giorno in giorno. Molti giovani si trovano ad affrontare, pertanto, un futuro incerto. La cooperativa offre loro un'alternativa basata su valori come la democrazia e la prevalenza della persona sul capitale.

In un mondo sempre più globalizzato, le cooperative offrono la loro capacità storica di ridurrere in modo equo le divisioni sociali ed economiche, almeno per coloro che hanno qualche possibilità di controllare le loro scelte. Come sempre hanno fatto in passato, le cooperative offrono I' opportunità alle persone di essere di aiuto a loro stessi. Infatti le cooperative contribuiscono allo sradicamento della povertà, generando occupazione sia questa diretta che indiretta.

Questo aspetto, che è stato essenziale per le origini e la nascita del movimento cooperativo, continua ad essere $d^{\prime}$ 'interesse vitale in questo particolare momento in cui il problema principale di molte economie continua è la disoccupazione. E questa opzione dovrebbe essere abbastanza attraente per affascinare i giovane nel momento in cui entrano nel mercato globale.

\subsection{La formazione e l'educazione dei giovani}

Un altro obiettivo del movimento cooperativo verso i giovani è volto alla loro formazione e alla loro istruzione. II movimento cooperativo appare come il mezzo ideale per educare i giovani perché forma ai valori umani di democrazia, di solidarietà e di altruismo. E, d'altra 
parte, una gioventù formata diviene una garanzia di efficacia per la cooperativa che integra.

$\mathrm{L}^{\prime} \mathrm{ACl}$, consapevole della mancanza di attrazione del movimento cooperativo tra i giovani, fin dagli anni sessanta predica la necessità di formare giovani, utilizzando forme di espressione ed di educazione adatti ai cambiamenti tecnologici, sociali e culturali della gioventù. Questa preoccupazione è stata a valorizzata nel quinto principio della Dichiarazione sull'Identità Cooperativa approvata dal Congresso dell'ACl di Manchester nel 1995, secondo il quale le cooperative informeranno al pubblico, soprattutto ai leader di opinione e ai giovani, della natura e dei benefici della cooperazione.

Tuttavia, la strada non è priva di ostacoli. Un gran numero dei giovani studenti dicono $d^{\prime}$ ignorare la realtà delle cooperative, anche se quella stessa maggioranza ritiene di dover introdurre questi argomenti nel piano di studio. È proprio l'ignoranza di questa figura giuridica una delle ragioni per cui i giovani imprenditori non scelgono le cooperative per iniziare un'attività economica. Forse qualche conoscenza sulle cooperative permetterebbe un aproccio più inmediato da parte dei giovani a maggior ragione che gran parte della gioventù condivide i valori di solidarietà, di democrazia e di partecipazione.

A nostro parere, nella realtà attuale stiamo assistendo ad una nuova sfida: affrontare la responsabilità delle cooperative, in modo singolare sollecitando il movimento nel suo complesso per quanto riguarda l'istruzione, la formazione e l'informazione delle nuove generazioni. Più che la responsabilità, abbiamo il dovere di fare vedere loro e spiegare la natura e i benefici della cooperazione.

In un altro caso, se sprechiamo l'opportunità che i giovani rappresentano e ignoriamo o trascuriamo la nostra responsabilità rispetto a loro, sarà molto difficile che possano sviluppare tutto il loro potenziale.

In questo senso ha proprio parlato I'Alleanza Cooperativa Internazionale, sottolineando le difficoltà che susistono. Da qui deriva l'importanza dell'istruzione e della formazione in valori e principi cooperativi come strumenti o come mezzo per rafforzare la sfida di attrarrere giovani verso il mondo cooperativo, per il consolidamento del movimento e il rinovamento generazionale.

Per fare questo, l'educazione cooperativa deve essere coraggiosa e così immaginativa, come nelle sue origini, accettando e adattandosi alle nuove opportunità che offrono le tecnologie innovative, rafforzando i legami tra università, ricerca e transferimento di conoscenza, al fine di informare e diffondere il suo messaggio. In definitiva, un'educazione cooperativa con successo deve adattarsi e non dimenticare mai lo 
scopo principale che è quello di sviluppare una maggiore comprensione della natura e dei benefici della cooperazione.

Siamo in conformità con il Professore MARTíNEZ CHARTERINA quando afferma che la presenza delle cooperative nella società e la loro aspirazione di conquista non può essere concepita senza che sia difusa la convinzione della bontà del sistema. Le cooperative essendo disposte ad ascoltare e ad adattarsi a le varie essigenze e a i vari bisogni devono essere in grado di inspirare e di attrarerre le nuove generazione.

Ci sono alcuni paesi che hanno favorito la creazione di cooperative di giovani studenti. Questa innovativa forma di apprendistato è stata applicata con successo in diversi paesi del mondo. Ad esempio, in Finlandia, hanno istituito le cooperative Learning Team, nei Paesi Baschi le Cooperative Junior. Nel caso del Paese Basco, è neccesario che la nuova azienda abbia la parola «junior» perchè essa possa essere considerata a tutti gli effetti una azienda reale. Per fare questo semplicemente basta che venga notificato al registro delle cooperative. Quando i giovani studenti concluderano il periodo di studio queste cooperative diventeranno cooperative di lavoro normale.

\subsection{La partecipazione dei giovani nella cooperativa}

Le cooperative affrontano anche un altro problema legato a i giovani: la mancanza di partecipazione e di accesso a ruoli di responsabilità, e trovano una soluzione attraverso il modo di concepire la participazione democratica dei soci nella azienda.

I giovani possono fissare obiettivi secondo le loro preoccupazioni e le loro competenze, così come possono partecipare al processo decisionale divenendo protagonisti di un progetto collettivo gestito democraticamente.

La partecipazione dei giovani alla direzione delle cooperative renderebbe le cooperative più attraenti perchè essi sapranno come migliorare quegli aspetti che sembrano a loro più interessanti,

ma allo stesso tempo, I'obiettivo resta sempre quello di educare i giovani alla responsabilità, esercitandola.

II movimento cooperativo vuole non cadere nel errore di creare cooperative rigorosamente composte da giovani, e così promuove l'integrazione globale con soci di qualsiasi età.

Così, ancora una volta, la democrazia acquisisce la sua pienezza come valore cooperativo, rendendo l'integrazione dei giovani un processo equo, così da evitare che le gerarchie di età tra i membri possano 
impedire l'integrazione e l'assunzione delle responsabilità per i più giovani.

Imparare dall'esperienza è ancora indispensabile per creare cooperative che possona funzionare nei nostri giorni. Solo attraverso programmi efficaci d'educazione cooperativa si può contribuire alla rinascita e al rinovamento del movimento cooperativo stabilito, così come per sblocare la visione e le energie delle nuove generazioni, rivelando come può essere applicato il modello cooperativo alle sfide di oggi e di domani.

In definitiva, usando le parole dell' $\mathrm{ACl}$, per ragioni sia economiche che di giustizia, le cooperative hanno l'obbligo di rivolgersi a i giovani costantemente e responsabilmente. II futuro del movimento cooperativo appartiene a i giovani. Le ricche e varie tradizioni del movimento, le sfumature e il potenziale della loro filosofia devono essere riconsiderati e riappliacati per ogni generazione. Quanto prima si coinvolgono i giovani, tanto prima essi iniziano a pensare come il movimento cooperativo dovrebbe addeguarsi ad ogni epoca. Solo così sarà meglio per tutti.

Pertanto, il dialogo attraverso le generazioni della cooperativa è un requisito fondamentale per il continuo successo delle stesse.

Grazie mille.

\section{Bibliografia}

ALIANZA COOPERATIVA INTERNACIONAL. Declaración de la Alianza Cooperativa Internacional sobre la identidad cooperativa. Los principios cooperativos, Consejo Superior de Cooperativas de Euskadi, Vitoria-Gasteiz, 1996.

ALONSO RODRIGO, E. Fiscalidad de cooperativas y sociedades laborales, Generalitat de Catalunya, Barcelona, 2001.

BANCEL, JL. «Tercer principio: contribución económica de los socios», en Guías orientativas de la Comisión de Principios de la ACl, p. 4, in http://ica. coop/sites/default/files/attachments/DRAFT\%20Guidance $\% 20$ Notes $\% 20$ P3\%2C\%20P5\%2C\%20P7\%20Spanish\%202014-02-11.pdf.

BAREA TEJEIRO, J. Economía Social e inserción laboral de las personas con discapacidad en el País Vasco, Fundación BBVA, Bilbao, 2008.

BIBBY SCULLION, J. "Pensar globalmente», en Julia SMITH, Robin PUGA ed lan MACPHERSON (eds.), Los jóvenes reinventan las cooperativas (traduzione in spagnolo da Mirta Vuotro), Prometeo, Buenos Aires, 2006, pp. 27-30.

BÖÖK, S.A. Valores para un mundo en cambio, ACl, San José de Costa Rica, 1992.

CASTAÑO COLOMER, J. «Conflictos respecto a la práctica de los principios y valores cooperativos: el caso de España», Anuario de Estudios Cooperativos, núm. 1, 1995, pp. 33-43. 
COLOMBAIN, M. Las cooperativas, Organización Internacional del Trabajo, Ginebra, 1972, 9. ${ }^{\text {a }}$ ed.

DAVIDOVIC, G. Hacia un mundo cooperativo, Federación Nacional de Cooperativas de España, Zaragoza, 1976.

DIVAR GARTEIZ-AURRECOA, J. Economía y democracia, Dykinson, Madrid, 2013.

DIVAR GARTEIZ-AURRECOA, J. Las cooperativas: una alternativa económica, Dykinson, Madrid, 2011.

DRIMER, B. e KAPLAN DE DRIMER, A. Las cooperativas: fundamentos, historia, doctrina, Buenos Aires, Intercoop, 1981, 3. ${ }^{\text {a ed. }}$

GADEA SOLER, E. «Prólogo», in DIVAR GARTEIZ-AURRECOA, J., Las cooperativas: una alternativa económica, Dykinson, Madrid, 2011, pp. 11-14.

GÓMEZ URQUIJO, L.T. La Alianza Cooperativa Internacional. Su desarrollo como institución y en especial como instrumento transformador de la sociedad, Consejo Superior de Cooperativas de Euskadi, Vitoria-Gasteiz, 1998.

MARCUS, L. "Cooperatives and Basic Values», ICA, XXIX Congress, Stockholm, July 1988, Agenda \& Reports, ICA, Geneva, 1988, pp. 95-108.

MARTÍNEZ CHARTERINA, A. "Los valores y los principios cooperativos», REVESCO, núm. 41, 1995, pp. 35-45.

MILLS, C. e DAVIES, W. Plan para una Década Cooperativa, ACI, 2013. Può essere trovato in http://ica.coop/sites/default/files/attachments/lCA\%20Blueprint\%20 -\%20Final\%20-\%20March\%2013\%20ES.pdf.

SMITH, J., PUGA, R. e MACPHERSON, I. (eds.). Los jóvenes reinventan las cooperativas (traduzione in spagnolo da Mirta VUOTTO), Prometeo, Buenos Aires, 2006. 